University of Nebraska - Lincoln

DigitalCommons@University of Nebraska - Lincoln

US Department of Energy Publications

U.S. Department of Energy

2017

\title{
Polymeric spatial resolution test patterns for mass spectrometry imaging using nano-thermal analysis with atomic force microscopy
}

Tamin Tai

Oak Ridge National Laboratory

Vilmos Kertesz

Oak Ridge National Laboratory

Ming-Wei Lin

Oak Ridge National Laboratory

Bernadeta R. Srijanto

Oak Ridge National Laboratory

Dale K. Hensley

Oak Ridge National Laboratory

See next page for additional authors

Follow this and additional works at: http:// digitalcommons.unl.edu/usdoepub

Tai, Tamin; Kertesz, Vilmos; Lin, Ming-Wei; Srijanto, Bernadeta R.; Hensley, Dale K.; Xiao, Kai; and Van Berkel, Gary J., "Polymeric spatial resolution test patterns for mass spectrometry imaging using nano-thermal analysis with atomic force microscopy" (2017). US Department of Energy Publications. 369.

http://digitalcommons.unl.edu/usdoepub/369

This Article is brought to you for free and open access by the U.S. Department of Energy at DigitalCommons@University of Nebraska - Lincoln. It has been accepted for inclusion in US Department of Energy Publications by an authorized administrator of DigitalCommons@University of Nebraska Lincoln. 
Authors

Tamin Tai, Vilmos Kertesz, Ming-Wei Lin, Bernadeta R. Srijanto, Dale K. Hensley, Kai Xiao, and Gary J. Van Berkel 


\title{
Polymeric spatial resolution test patterns for mass spectrometry imaging using nano-thermal analysis with atomic force microscopy
}

\author{
Tamin Tai ${ }^{1 \dagger}$, Vilmos Kertesz ${ }^{1}$, Ming-Wei Lin², Bernadeta R. Srijanto ${ }^{2}$, Dale K. Hensley ${ }^{2}$, \\ Kai Xiao ${ }^{2}$ and Gary J. Van Berkel ${ }^{1 *}$ \\ ${ }^{1}$ Mass Spectrometry and Laser Spectroscopy Group, Chemical Sciences Division, Oak Ridge National Laboratory, Oak Ridge, \\ TN 37831, USA \\ ${ }^{2}$ Center for Nanophase Materials Sciences, Oak Ridge National Laboratory, Oak Ridge, TN 37831, USA
}

RATIONALE: As the spatial resolution of mass spectrometry imaging technologies has begun to reach into the nanometer regime, finding readily available or easily made resolution reference materials has become particularly challenging for molecular imaging purposes. This paper describes the fabrication, characterization and use of vertical line array polymeric spatial resolution test patterns for nano-thermal analysis/atomic force microscopy/mass spectrometry chemical imaging. METHODS: Test patterns of varied line width $(0.7$ or $1.0 \mu \mathrm{m})$ and spacing $(0.7$ or $1.0 \mu \mathrm{m})$ were created in an $\sim 1-\mu \mathrm{m}$-thick poly(methyl methacrylate) thin film using electron beam lithography. The patterns were characterized by scanning electron microscopy, energy-dispersive X-ray spectroscopy, atomic force microscopy topography and nano-thermal analysis/mass spectrometry imaging.

RESULTS: The efficacy of these polymeric test patterns for the advancement of chemical imaging techniques was illustrated by their use to judge the spatial resolution improvement achieved by heating the ionization interface of the current instrument platform. The spatial resolution of the mass spectral chemical images was estimated to be $1.4 \mu \mathrm{m}$, based on the ability to statistically distinguish $0.7-\mu \mathrm{m}$-wide lines separated by $0.7-\mu \mathrm{m}$-wide spacings in those images when the interface cross was heated to $200^{\circ} \mathrm{C}$.

CONCLUSIONS: This work illustrates that e-beam lithography is a viable method to create spatial resolution test patterns in a thin film of high molecular weight polymer to allow unbiased judgment of intra-laboratory advancement and/or inter-laboratory comparison of instrument advances in nano-thermal analysis/atomic force microscopy/mass spectrometry chemical imaging. Published in 2017. This article is a U.S. Government work and is in the public domain in the USA.

Mass spectrometry imaging (MSI) is used to visualize the spatial distribution of elemental or molecular species at or near a sample surface. ${ }^{[1]}$ A large variety of MSI approaches exist and many are rapidly advancing in performance, particularly spatial resolution. In order to test and evaluate the advances in these instruments with respect to spatial resolution, both the appropriate resolution reference materials and unbiased procedures to evaluate the quality of the images produced are required. As the spatial resolution of these technologies has begun to reach into the nanometer regime, finding readily available or easily made resolution reference materials has become particularly challenging for both elemental ${ }^{[2]}$ and molecular imaging purposes. ${ }^{[3-5]}$

\footnotetext{
* Correspondence to: G. J. Van Berkel, Mass Spectrometry and Laser Spectroscopy Group, Chemical Sciences Division, Oak Ridge National Laboratory, Oak Ridge, TN 37831, USA.

E-mail: vanberkelgj@ornl.gov

+ Present address: Laboratory for Physical Sciences, 8050 Greenmead Drive, College Park, MD 20740, USA.
}

In the MSI world of secondary ionization mass spectrometry (SIMS) and laser ablation-inductively coupled plasma mass spectrometry(LA-ICP/MS), where surface sampling is by particle bombardment or LA and elemental analysis is performed, suitable certified resolution reference materials exist. $^{[2,6,7]}$ Moreover, creating reference materials of various metals using traditional microfabrication techniques such as metalorganic vapor phase epitaxy growth ${ }^{[8]}$ and molecular beam epitaxy ${ }^{[9]}$ is a relatively straightforward process. For organic and biological MSI, where (intact) molecular species are sampled and detected, the availability and use of spatial resolution reference materials are not so advanced. Complicating the issue is the fact that the nature of the sampling process (e.g., laser ablation, liquid extraction, thermal desorption) and intertwined (e.g., matrix-assisted laser desorption/ionization (MALDI)) or separate ionization processes (e.g., electrospray ionization (ESI) or atmospheric pressure chemical ionization (APCI)) may necessitate a specific reference standard appropriate for each particular type of MSI system. Some typical resolution standards that have been reported in the literature include stamped ink patterns ${ }^{[10-12]}$ ink jet patterns printed using 'ink' containing a suitable test compound, ${ }^{[13-16]}$ analyte surfaces covered by 
masking grids ${ }^{[5,17]}$ patterns in photoresist materials ${ }^{[18,19]}$ and microfabricated surface wells and patterns filled, covered or functionalized with organic or biological molecules. ${ }^{[3-5]}$

One MSI approach that we are advancing is based on an ambient environment nanoscale thermal desorption, or a thermolysis, sampling process using nano-thermal analysis (TA) probes controlled with an atomic force microscope. ${ }^{\text {[20-23] }}$ In its simplest form, a nano-TA probe is heated to the appropriate temperature and placed in close proximity to or in actual contact with the sample surface. This method results in the local desorption of intact molecular species or liberates into the gas-phase products of the thermolysis of the surface materials. These gas-phase species are then ionized under atmospheric pressure conditions by ESI ${ }^{[20]}$ or $\mathrm{APCI}^{[21-23]}$ and analyzed using MS.

To date, the imaging spatial resolution test materials used with this system have been either inkjet-printed grid patterns ${ }^{[20]}$ or phase-separated polymer thin films. ${ }^{[2,23]}$ Using a phase-separated poly(2-vinylpyridine) (P2VP)/poly(methyl methacrylate) (PMMA) thin film, we were able to demonstrate a spatial imaging resolution of the nano-thermal imaging/ atomic force microscopy/mass spectrometry (TA/AFM/MS) imaging system of about $1.6 \mu \mathrm{m} .{ }^{[23]}$ This resolution limit was based on the ability to distinguish particular adventitious chemical features of this size in the polymer sample that were visible in both the MS-based image and the AFM-based topographical image of the sample. As we have continued to advance the capabilities of the nano-TA/AFM/MS imaging system for polymer thin film analysis, it became evident that a resolution reference material with similar or even smaller sized features, that could be reliably reproduced and characterized by both the nano-TA/AFM/MS system and independent methods, would be required for unbiased judgment of imaging capability/quality of these films.

To that end, we describe here the fabrication, characterization and use of polymeric vertical line array spatial resolution test patterns of varied line widths and spacings created in a 1- $\mu \mathrm{m}$-thick PMMA film. The general utility of these test patterns is demonstrated by their use in evaluating the changing mass spectral image quality achieved as a function of the ionization interface temperature on our current nano-TA AFM/MS system. The mass spectra spatial resolution was evaluated in a non-biased, quantitative fashion using the recently developed metric "corrected resolving power factor" (cRPF). ${ }^{[19]}$ The best imaging resolution for the given PMMA thin film, achieved with the heated interface, was estimated to be $1.4 \mu \mathrm{m}$ based on the ability to statistically distinguish $0.7-\mu \mathrm{m}$-wide lines separated by $0.7-\mu \mathrm{m}$-wide spacings.

\section{EXPERIMENTAL}

Samples and solvents, polymer thin film preparation, fabrication of PMMA resolution targets, scanning electron microscope (SEM) and energy-dispersive $X$-ray spectroscopy (EDX) characterizations of PMMA resolution targets

The materials used and these processes are described in detail in the Supporting Information.

\section{Nano-TA/AFM/MS system and imaging procedure}

The basic nano-TA/AFM/MS system is shown in Fig. 1 and has been described in detail elsewhere. ${ }^{[22]}$ The AFM instrument used here was a Veeco Multimode (Bruker AXS, Santa Barbara, CA, USA) equipped with a closed loop stage from N-Point (Madison, WI, USA) and a Nanonis system controller (SPECS Zurich GmbH, Zurich, Switzerland). The heated tips were VITA-MM-NANOTA300 nano-TA AFM probes (Bruker AXS, Camarillo, CA, USA). For imaging experiments, the AFM instrument was controlled using custom software that utilized components of the Nanonis interface and in-house developed software. This custom software modified the nano-TA probe heating voltage on each line scan trace $(10 \mathrm{~V}$, thermolysis at $\left.>370^{\circ} \mathrm{C}\right)$ and retrace $\left(3.5 \mathrm{~V},<100^{\circ} \mathrm{C}\right.$ to minimize heatinduced mechanical deformation of the cantilever). The mass spectrometer used was a LTQ XL linear ion trap (Thermo Fisher Scientific, Waltham, MA, USA) operated in positive ion mode. Material liberated from the surface by thermolysis at the AFM nano-TA probes was drawn into an in-line vapor extractor/corona discharge APCI source via a 2.5cm-long extractor capillary $(0.85 \mathrm{~mm}$ o.d. $\times 0.50 \mathrm{~mm}$ i.d.) placed next to the AFM probe. The temperature of this 'cross' ionization region was a controlled variable. Ions created in this region were transferred into the mass spectrometer for analysis. Protonated methyl methacrylate from the thermolysis of PMMA was detected via tandem mass spectrometry $(\mathrm{m} / \mathrm{z} 101 \rightarrow 73$, normalized collision energy (CE) of 35\%). Lane scan speed and lane spacing were selected such that the horizontal pixel dimensions matched the lane spacings, resulting in square-shaped mass spectral data pixels $(330 \mathrm{~nm} \times 330 \mathrm{~nm})$ for every image acquired. The 2D MS images shown in the text were generated by the in-house developed software package MultiDimChemImaging.

\section{RESULTS AND DISCUSSION}

\section{Resolution test patterns}

Resolution test patterns for the nano-TA AFM/MS system were fabricated from a spin-coated high molecular weight PMMA thin film ( 1 $\mu \mathrm{m}$ thick) using electron beam lithography (EBL). High molecular weight PMMA is among a family of vinyl-linked polymers known to quantitatively 'unzip' to the monomer, in this case methyl methacrylate, ${ }^{[24]}$ upon heating to a suitable temperature. Methyl methacrylate is a volatile compound that can be ionized and detected intact as the protonated molecule by positive ion APCI-MS. Moreover, this polymer film and patterns created therein were found to be stable under ambient condition for extended periods (at least several weeks). The vertical line array test patterns created were a series of six rectangular trenches extending through the polymer all the way to the silicon wafer substrate, each with a length of $10 \mu \mathrm{m}$ and line widths/line spacing of $0.7 / 0.7,0.7 / 1.0$ or $1.0 / 1.0 \mu \mathrm{m}$ (Fig. 2). Pattern integrity was confirmed by SEM (Figs. 2(a), 2(g), and $2(\mathrm{~m}))$, while EDX of the patterns confirmed the complete removal of the polymer film from the trenches (Figs. 2(b), 2(h), and 2(n)). 


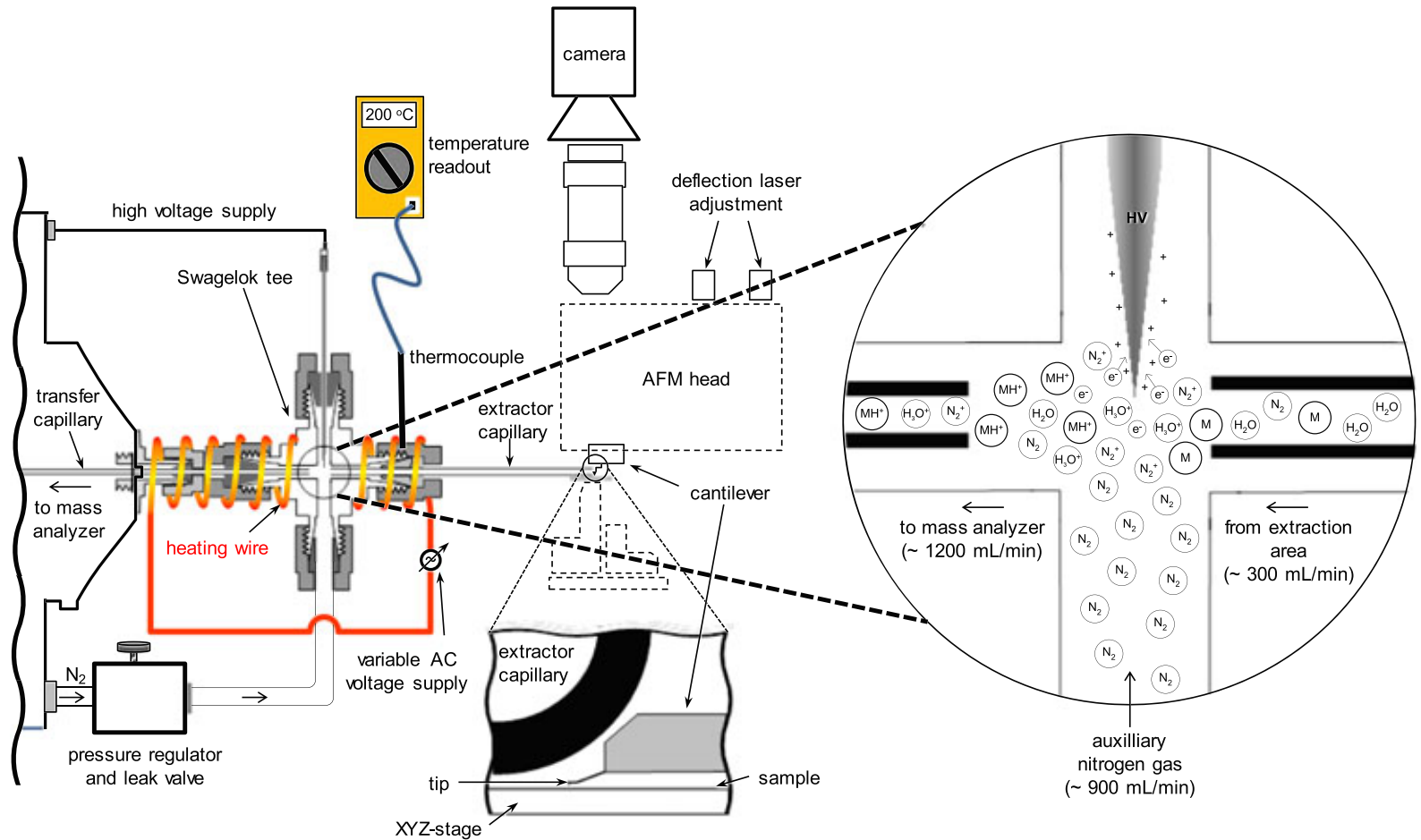

Figure 1. Schematic illustration of the nano-TA/AFM/MS experimental setup with an enlarged view showing the details of the inline APCI and ion molecule chemistry and an enlarged view of the AFM nano-TA probe positioned $<0.5 \mathrm{~mm}$ away from the sampling capillary. A variac-controlled heating wire and a thermocouple were used to vary and measure the temperature of the interface cross. [Color figure can be viewed at wileyonlinelibrary.com]

\section{Nano-TA/AFM/MS chemical imaging spatial resolution as a function of interface cross temperature}

The nano-TA AFM-MS system utilizes the same cantilever probe to serially acquire topographic and chemical images of the sample surface. The images in Figs. 2(c), 2(i), and 2(o) are the AFM topographic images of the three different resolution test patterns, which match well with the SEM images. The mass spectral chemical imaging of the resolution test patterns was carried out using the same nano-TA cantilever probe but in this case at a temperature in excess of about $370^{\circ} \mathrm{C}$. When contacted by the probe at this temperature, the backbone of the PMMA polymer unzipped releasing into the gas phase the methyl methacrylate monomer (see Supplementary Scheme S1, Supporting Information). The gas-phase monomers were drawn by the vacuum pull of the mass spectrometer into an extractor capillary and on into an incapillary corona discharge APCI source, ${ }^{[25]}$ then into the mass spectrometer. As such, the major ion-molecule reaction in positive ion mode with the vapor material sampled was expected to be proton transfer resulting in the formation of protonated monomer molecules $[\mathrm{M}+\mathrm{H}]^{+}$, and protonated methyl methacrylate was observed at $m / z 101$. However, using MS/MS of the protonated molecule $(\mathrm{m} / \mathrm{z} 101 \rightarrow 73$, see Supplementary Scheme S1, Supporting Information) added detection specificity, because of background chemical noise in this region of the mass spectrum. The signal intensity recorded at each specific location (mass spectral data pixel) during a series of unidirectional line scans was used to render the mass spectral chemical images.
The temperature of the interface cross, where transport and ionization of neutrals from the nano-TA desorption/ thermolysis process take place, might be expected to be an important experimental parameter influencing the observed signal and thus the mass spectral image quality. Depending on the nature of the chemical species involved, materials may be lost from the gas phase to cool surfaces along the transport path to the ionization region. We in fact found that the signal-to-noise $(S / N)$ levels for the analysis of methyl methacrylate from the thermolysis of PMMA increased with increasing temperature from ambient up to about $200^{\circ} \mathrm{C}$. Those experiments used an $\sim 1-\mu$ m-thick PMMA film that did not contain the test patterns. The $S / N$ was calculated using mass-to-charge chronograms of methyl methacrylate collected during thermolysis and that collected while scanning over the PMMA surface with no voltage (no heat) applied to the AFM tip. The data in Fig. 3(a) shows that there was about a $4 \times$ improvement in $S / N$ in the signal from the PMMA monomer when the temperature of the interface cross $\left(T_{i c}\right)$ was increased from ambient (approximately $30^{\circ} \mathrm{C}$ due to the heated transfer capillary between the cross and the mass spectrometer inlet) to $200^{\circ} \mathrm{C}$. Corresponding background-subtracted product ion mass spectra of $\mathrm{m} / \mathrm{z}$ 101 at $T_{i c}=30^{\circ} \mathrm{C}$ and $T_{i c}=200^{\circ} \mathrm{C}$ are shown in Figs. 3(b) and $3(\mathrm{c})$, respectively. Note that the temperature of the extractor capillary positioned close to the sample was only elevated slightly above ambient by this heating process. Within just $5 \mathrm{~mm}$ out from the cross, which was actively heated by a heating wire, the extractor capillary temperature dropped to $35^{\circ} \mathrm{C}$. 

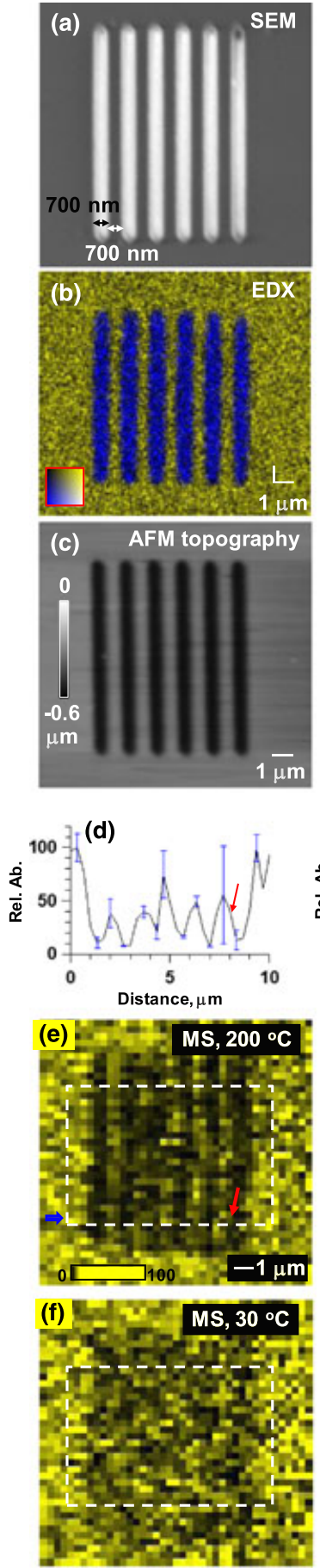
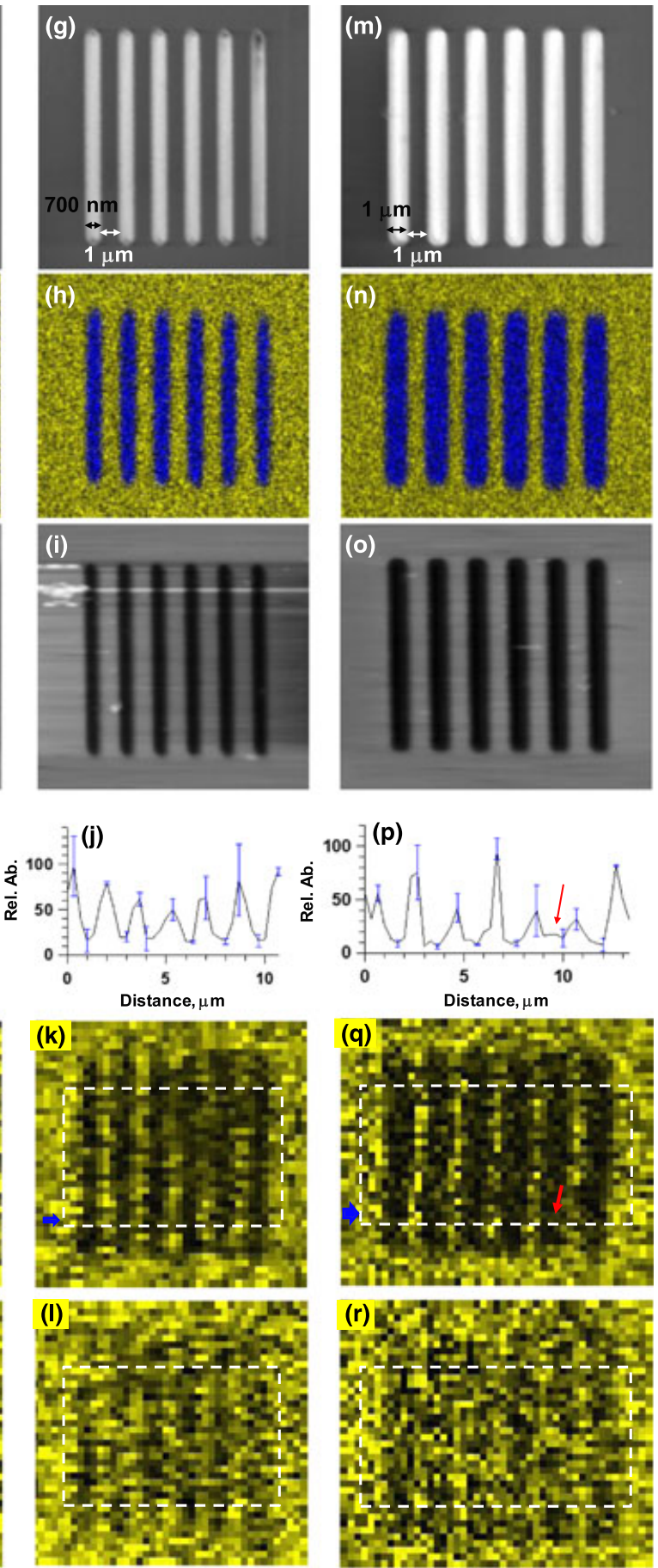

Figure 2. (a, g, m) SEM topographical, (b, h, n) EDX elemental (C: yellow, Si: blue), (c, i, o) AFM topographical (with height scale in (c)), and mass spectral chemical images $(0.33 \mu \mathrm{m}$ square pixel size) acquired at an interface cross temperature of $(\mathrm{e}, \mathrm{k}, \mathrm{q}) \sim 200^{\circ} \mathrm{C}$ and $(\mathrm{f}, \mathrm{l}, \mathrm{r}) \sim 30^{\circ} \mathrm{C}$ for spatial resolution test patterns with $0.7 / 0.7$, $0.7 / 1.0$ and 1.0/1.0 $\mu \mathrm{m}$ line widths/line spacings, respectively. Calculated average intensities of the bottom $(d, j)$ two or $(p)$ three lanes (pointed out by blue arrows) acquired for areas indicated by white dashed rectangles in the chemical images acquired at an interface cross temperature of $\sim 200^{\circ} \mathrm{C}$ for the $0.7 / 0.7,0.7$ / 1.0 and $1.0 / 1.0 \mu \mathrm{m}$ patterns in $(\mathrm{e}, \mathrm{k}, \mathrm{q})$, respectively. Distances shown were measured from the beginning of the sections and the standard deviations calculated from signal statistics are shown at the peaks and valleys. Red arrows in (d) and (p) indicate areas in which the standard deviations of the peaks and valleys overlap. Areas indicated by white dashed rectangles in (e, k, q) and (f, l, r) were used for the statistical analysis presented in Supplementary Fig. S1 (Supporting Information). [Color figure can be viewed at wileyonlinelibrary.com] 

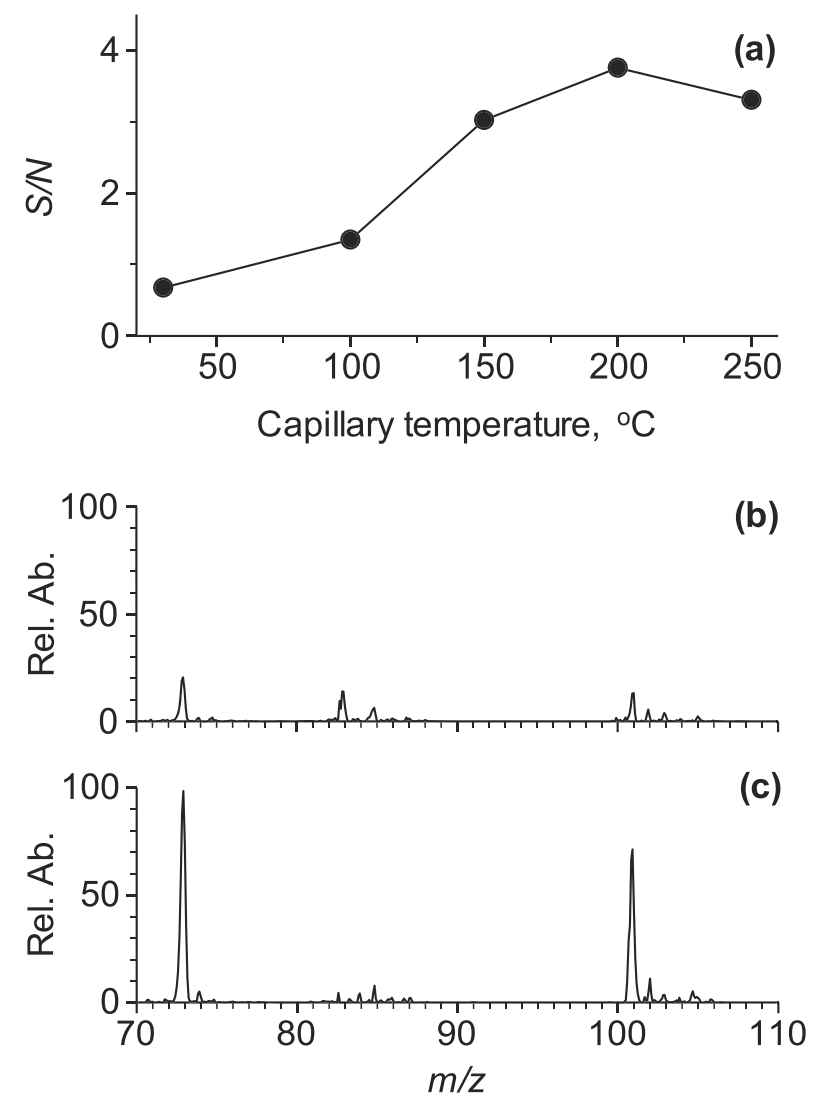

Figure 3. (a) Mass spectral $S / N$ levels calculated for the AFM nano-TA/AFM/MS of a $10 \mu \mathrm{m} \times 10 \mu \mathrm{m}$ area of a $\sim 1$ - $\mu \mathrm{m}$-thick PMMA film scanned at $2.4 \mu \mathrm{m} / \mathrm{s}$ with $0.5 \mu \mathrm{m}$ lane spacing $(0.5 \mu \mathrm{m}$ square pixel) as a function of APCI interface cross temperature. Background-subtracted product ion mass spectra of $m / z 101$ using interface cross temperatures of (b) $30^{\circ} \mathrm{C}$ and (c) $200^{\circ} \mathrm{C}$. Signal levels in (b) normalized to the maximum signal in (c).

Figures 2(e), 2(k) and 2(q), and 2(f), 2(l) and 2(r), show the mass spectral chemical images for these test patterns obtained with the interface cross at $200^{\circ} \mathrm{C}$ and $30^{\circ} \mathrm{C}$, respectively. For this data set, visual observation of the different test pattern images revealed that heating the interface cross improved image quality. At ambient temperature it was very difficult to visually discern the vertical line array of the test pattern in any of the three images. However, patterns were visible when the interface cross temperature was $200^{\circ} \mathrm{C}$. To ensure a non-biased judgement, the mass spectra spatial resolution of the images was evaluated using the recently developed metric "corrected resolving power factor" (cRPF). ${ }^{[19]}$ Mass-tocharge chronograms of methyl methacrylate for 21, 330-nm-wide lanes (corresponding to a total vertical width of $\sim 7 \mu \mathrm{m}$ ) across the center of the region that should have contained the five vertical line arrays in the chemical images (areas indicated by dashed white lines in Figs. 2(e), 2(f), 2(k), 2(l), 2(q), and 2(r)) were used for the calculation of the individual $c R P F$ values. The number of lines statistically analyzed simultaneously corresponded to the feature size(s) investigated so as not to bias the calculations towards higher spatial resolutions and to report spatial resolutions as accurately as possible. ${ }^{[19]}$ For this reason, all possible consecutive two- and three-line-wide bands (corresponding to total band widths of $660 \mathrm{~nm}$ and $990 \mathrm{~nm}$, respectively) were used to evaluate patterns with $700 \mathrm{~nm}$ and $1000 \mathrm{~nm}$ diameters, respectively. To illustrate this process, Figs. 2(d), 2(j) and 2(p) show calculated average intensities of the bottom two- and three-line-wide bands in the white dashed rectangles shown in Figs. 2(e), $2(\mathrm{k})$ and 2(q), respectively, as a function of distance from the start of those rectangles. In addition, the standard deviations calculated from signal statistics are shown at the peaks and valleys. The presence or absence of overlap between adjacent standard deviation values indicates if a feature was resolved or not (red arrows in Figs. 2(d) and 2(p) indicate areas in which adjacent standard deviation values did in fact overlap), respectively, by definition of ${ }_{c R P F}{ }^{[19]}$ (Signal intensity asymmetry, i.e. signal did not increase to its maximum level when the probe scanned over the lines, was probably because not all of the material was liberated to the gas phase; some was redistributed, similarly to other imaging techniques such e.g. laser ablation-based techniques (material redeposition) and desorption electrospray ionization (DESI) (analyte is rearranged on the surface) $).{ }^{[26]}$ ) Repeating this process for areas indicated by dashed white rectangles in Figs. 2(e), $2(\mathrm{k})$ and 2(q) resulted in 100 (i.e., 20 different two-linewide lanes analyzed for five vertical array lines) and 95 (i.e., 19 different three-line-wide lanes analyzed for five vertical array lines) individual $c R P F$ values for patterns with $700 \mathrm{~nm}$ and $1000 \mathrm{~nm}$ diameters, respectively.

The fraction of cases where the features were resolved in this data analysis (i.e. when $c R P F>0$ ) are shown in Supplementary Fig. S1(a) (Supporting Information) for the different PMMA patterns, viz., 0.7/0.7, 0.7/1.0 and 1.0/1.0 $\mu \mathrm{m}$ line width/line spacings, respectively, using a $T_{i c}$ of (hatched bars) $30^{\circ} \mathrm{C}$ and (crosshatched bars) $200^{\circ} \mathrm{C}$. These data demonstrate that in $70-80 \%$ of cases, the featured vertical line arrays were resolved using $T_{i c}=200^{\circ} \mathrm{C}$, while $\leq 10 \%$ were resolved employing $T_{i c}=30^{\circ} \mathrm{C}$. To further investigate the statistical significance of this low success rate at $T_{i c}=30^{\circ} \mathrm{C}$, chemical images were generated by in-house software using pixels with random intensities (empty bars plots in Supplementary Fig. S1, see Supporting Information for more details). The ratios of successfully resolved features using these random chemical images are also shown in Supplementary Fig. S1(a) (Supporting Information) and in all cases they were $\leq 7 \%$. This means that chemical images acquired with $T_{i c}=30^{\circ} \mathrm{C}$ show only a weak statistical advantage over the randomly generated chemical images with regard to resolving 700 and $1000 \mathrm{~nm}$ features. The vertical arrays of lines in the test patterns were not resolved. In addition, it also means that these vertical arrays of lines in the test patterns were resolved with great statistical probability when the chemical images were acquired with $T_{i c}=200^{\circ} \mathrm{C}$.

This conclusion is further supported by additional statistical analysis of the successfully resolved features (i.e. when $c R P F>0$ ). The values and error bars in Supplementary Fig. S1(b) (Supporting Information) represent the average and standard deviation, respectively, of these $c R P F$ positive values. Data in this figure represent a higher $c R P F$ average (-cRPF of $\sim 0.4-0.5)$ for chemical images acquired with $T_{i c}=200^{\circ} \mathrm{C}$, than for those acquired with $T_{i c}=30^{\circ} \mathrm{C}$ or generated randomly ( $-c R P F$ of $\sim 0.2-0.3$ in both cases). In 
summary, the $700 \mathrm{~nm}$ features were resolved in the mass spectral chemical images in a statistically significant manner when the interface cross was heated to $200^{\circ} \mathrm{C}$.

\section{CONCLUSIONS}

In this paper, we have described vertical line array spatial resolution test patterns for nano-TA/AFM-MS chemical imaging fabricated by standard e-beam lithography methods in a thin film of high molecular weight PMMA. The resolution pattern included trenches of given width and frequency in the thin film. In the present case, we have only created three test patterns that we used to judge the spatial resolution of the nano-TA/AFM-MS system. However, current EBL systems can produce linewidths of the order of $10 \mathrm{~nm}$ or smaller. ${ }^{[27]}$ Thus, this approach and created patterns are well suited to evaluate molecular imaging resolution for thin polymer films in the low micrometer/submicrometer range for techniques capable of chemical imaging of such films. The availability of such standard test patterns allows unbiased judgment of intra-laboratory advancement and/or inter-laboratory comparison of instrument advances in chemical imaging of thin polymer films. However, the user has to keep in mind that, unlike the organic patterns created on flat surfaces by other groups, ${ }^{[3-5]}$ the patterns created here introduce a height variation into the sample surface. While having $\sim 1-\mu \mathrm{m}$-deep void areas in the sample is not a problem for the present nano-TA/AFM combination due to the nature of sampling (in-contact thermolysis), this variation in sample topology may introduce undesired signal variation using other sampling methods (e.g. laser focus changes during laser ablation sampling) and thus make these patterns inapplicable for these techniques. One possible solution for this problem would be to 'fill up' the voids with a second polymer that is immiscible with the first one (e.g. such as a water-soluble polymer).

The use of the created patterns was illustrated by the quantitative comparison of mass spectral image quality from these standard patterns as a function of ionization source interface cross temperature. The spatial resolution of the mass spectral chemical images was evaluated in a non-biased, quantitative fashion and it was estimated to be $1.4 \mu \mathrm{m}$ based on the ability to statistically distinguish $0.7-\mu \mathrm{m}$-wide lines separated by $0.7-\mu \mathrm{m}$-wide spacings in those images that were also observed in the SEM, EDX and AFM topography images of the same surface. Given that test patterns with much smaller features could also be prepared by EBL and mass spectrometers with about $\sim 100 \times$ more sensitivity exist on the market compared with our current instrumentation, ${ }^{[28,29]}$ it is envisioned that these patterns could also be used to advance the nano-TA-AFM/MS and/or laser ablation-based imaging methods ${ }^{[18]}$ into the nanometer scale spatial resolution capability of thin polymer films. The findings also suggest that in order to obtain the best spatial resolution for a phaseseparated polymer film, the compound-specific dependence of chemical image resolution/quality on the temperature of the interface cross should always be individually optimized. Data in the present paper suggest that this optimization would allow for smaller than $\sim 1.6 \mu \mathrm{m}$ features to be resolved in the substrates that were presented in our previous publication on nano-TA/AFM/MS imaging of a phase-separated thin polymer film. ${ }^{[23]}$

\section{Acknowledgements}

The work of T.T., V.K. and G.J.V.B. on the fundamentals, optimization, and application of the AFM/MS system was supported by the United States Department of Energy, Office of Science, Basic Energy Sciences, Chemical Sciences, Geosciences, and Biosciences Division. The e-beam fabrication (M.W.L. and K.X. supported by a Laboratory Directed Research and Development grant at Oak Ridge National Laboratory) and the SEM/EDX analysis (B.R.S. and D.K.H.) were performed at Center for Nanophase Materials Sciences, which is a DOE Office of Science User Facility and sponsored at Oak Ridge National Laboratory by the Scientific User Facilities Division, Office of Basic Energy Sciences, U.S. Department of Energy. This manuscript has been authored by UT-Battelle, LLC under Contract No. DE-AC05-00OR22725 with the U.S. Department of Energy. The United States Government retains and the publisher, by accepting the article for publication, acknowledges that the United States Government retains a non-exclusive, paid-up, irrevocable, world-wide license to publish or reproduce the published form of this manuscript, or allow others to do so, for United States Government purposes. The Department of Energy will provide public access to these results of federally sponsored research in accordance with the DOE Public Access Plan (http://energy.gov/downloads/doepublic-access-plan).

\section{REFERENCES}

[1] A. Bodzon-Kulakowska, P. Suder. Imaging mass spectrometry: Instrumentation, applications, and combination with other visualization techniques. Mass Spectrom. Rev. 2016, 35, 147.

[2] M. Senoner, A. Maaßdorf, H. Rooch, W. Österle, M. Malcher, M. Schmidt, F. Kollmer, D. Paul, V.-D. Hodoroaba, S. Rades, W.E. S. Unger. Lateral resolution of nanoscaled images delivered by surfaceanalytical instrument: Application of the BAM-L200 Certified Reference Material and related ISO standards. Anal. Bioanal. Chem. 2015, 407, 3211.

[3] M. K. Passarelli, J. Wang, A. S. Mohammadi, R. Trouillon, I. Gilmore, A. G. Ewing. Development of an organic lateral resolution test device for imaging mass spectrometry. Anal. Chem. 2014, 86, 9473.

[4] F. Zubair, B. M. Prentice, J. L. Norris, P. E. Laibinis, R. M. Caprioli. Standard reticle slide to objectively evaluate spatial resolution and instrument performance in imaging mass spectrometry. Anal. Chem. 2016, 88, 7302.

[5] S. R. Fagerer, A. Römpp, K. Jefimovs, R. Brönnimann, G. Hayenga, R. F. Steinhoff, J. Krismer, M. Pabst, A. J. Ibanez, R. Zenobi. Resolution pattern for mass spectrometry imaging. Rapid Commun. Mass Spectrom. 2015, 29, 1019.

[6] M. Senoner, T. Wirth, W. E. S. Unger. Imaging surface analysis: Lateral resolution and its relation to contrast and noise. J. Anal. At. Spectrom. 2010, 25, 1440.

[7] http://www.bam.de/de/rm-certificates_media/rm_cert_ layer_and_surface/bam_1200repe.pdf.

[8] I. M. Watson. Metal organic vapour phase epitaxy of AlN, GaN, InN and their alloys: A key chemical technology for advanced device applications. Coord. Chem. Rev. 2013, 257, 2120. 
[9] J.-P. Peng, J.-Q. Guan, H.-M. Zhang, C.-L. Song, L. Wang, K. He, Q.-K. Xue, X.-C. Ma. Molecular beam epitaxy growth and scanning tunneling microscopy study of $\mathrm{TiSe}_{2}$ ultrathin films. Phys. Rev. B 2015, 91, 121113.

[10] J. Laskin, B. S. Heath, P. J. Roach, L. Cazares, O. J. Semmes. Tissue imaging using nanospray desorption electrospray ionization mass spectrometry. Anal. Chem. 2012, 84, 141.

[11] O. S. Ovchinnikova, K. Kjoller, G. B. Hurst, D. A. Pelletier, G. J. Van Berkel. Atomic force microscope controlled topographical imaging and proximal probe thermal desorption/ionization mass spectrometry imaging. Anal. Chem. 2014, 86, 1083.

[12] O.S. Ovchinnikova, D. Bhandari, M. Lorenz, G. J. Van Berkel. Transmission geometry laser ablation into a non-contact liquid vortex capture probe for mass spectrometry imaging. Rapid Commun. Mass Spectrom. 2014, 28, 1665.

[13] D. R. Ifa, J. M. Wiseman, Q. Song, R. G. Cooks. Development of capabilities for imaging mass spectrometry under ambient conditions with desorption electrospray ionization (DESI). Int. J. Mass Spectrom. 2007, 259, 8.

[14] V. Kertesz, G. J. Van Berkel. Improved imaging resolution in desorption electrospray ionization mass spectrometry. Rapid Commun. Mass Spectrom. 2008, 22, 2639.

[15] J. T. Shelley, S. J. Ray, G. M. Hieftje. Laser ablation coupled to a flowing atmospheric pressure afterglow for ambient mass spectral imaging. Anal. Chem. 2008, 80, 8308.

[16] J. T. van Elteren, A. Izmer, V. S. Šelih, F. Vanhaecke. Novel image metrics for retrieval of the lateral resolution in line scan-based 2D LA-ICPMS imaging via an experimentalmodeling approach. Anal. Chem. 2016, 88, 7413.

[17] M. Koestler, D. Kirsch, A. Hester, A. Leisner, S. Guenther, B. Spengler. A high resolution scanning microprobe matrix-assisted laser desorption/ionization ion source for imaging analysis on an ion trap/Fourier transform ion cyclotron resonance mass spectrometer. Rapid Commun. Mass Spectrom. 2008, 22, 3275.

[18] J. F. Cahill, V. Kertesz, G. J. Van Berkel. Characterization and application of a hybrid optical microscopy/laser ablation liquid vortex capture/electrospray ionization system for mass spectrometry imaging with sub-micrometer spatial resolution. Anal. Chem. 2015, 87, 11113.

[19] V. Kertesz, J. F. Cahill, G. J. Van Berkel. Quantitative metrics for assessment of chemical image quality and spatial resolution. Rapid Commun. Mass Spectrom. 2016, 30, 927.

[20] O.S. Ovchinnikova, M. P. Nikiforov, J. A. Bradshaw, S. Jesse, G. J. Van Berkel. Combined atomic force microscope-based topographical imaging and nanometer-scale resolved proximal probe thermal desorption/electrospray ionization-mass spectrometry. ACSNano 2011, 5, 5526.

[21] O. S. Ovchinnikova, K. Kjoller, G. B. Hurst, D. A. Pelletier, G. J. Van Berkel. Atomic force microscope controlled topographical imaging and proximal probe thermal desorption/ionization mass spectrometry imaging. Anal. Chem. 2014, 86, 1083.

[22] O. S. Ovchinnikova, T. Tai, V. Bocharova, M. B. Okatan, A. Belianinov, V. Kertesz, S. Jesse, G. J. Van Berkel. Coregistered topographical, band excitation nanomechanical and mass spectral imaging using a combined atomic force microscopy/mass spectrometry platform. ACSNano 2015, 9,4260 .

[23] T. Tai, O. Karácsony, V. Bocharova, G. J. Van Berkel, V. Kertesz. Topographical and chemical imaging of a phase separated polymer using a combined atomic force microscopy/infrared spectroscopy/mass spectrometry platform. Anal. Chem. 2016, 88, 2864.

[24] C. L. Beyler, M. M. Hirschler. Thermal decomposition of polymers, in SFPE Handbook of Fire Protection Engineering, (3rd edn.), (Ed: P. J. DiNenno). NFPA, Quincy, MA, 2002, pp. 110-131.

[25] M. Moini. Atmospheric pressure chemical ionization: principles, instrumentation and applications, in The Encyclopedia of Mass Spectrometry, vol. 6, Ionization Methods, (Eds: M. L. Gross, R. M. Caprioli). Elsevier, New York, 2007, pp. 344-354.

[26] V. Kertesz, G. J. Van Berkel. Scanning and surface alignment considerations in chemical imaging with desorption electrospray mass spectrometry. Anal. Chem. 2008, 80, 1027.

[27] M. A. Mohammad, M. Muhammad, S. K. Dew, M. Stepanova. Fundamentals of electron beam exposure and development, in Nanofabrication, (Eds: M. Stepanova, S. Dew). SpringerVerlag, Vienna, Austria, 2012, pp. 11-41.

[28] http://tools.thermofisher.com/content/sfs/brochures/ PSB-128-Improved-Sensitivity-Through-Enhanced-IonTransmission-Using-an-S-Lens.pdf.

[29] L. Sun, G. Zhu, N. J. Dovichi. Comparison of LTQ-Orbitrap Velos and Q-Exactive for proteomic analysis of 1-1000 ng raw 264.7 cell lysate digests. Rapid Commun. Mass Spectrom. 2013, 27, 157.

\section{SUPPORTING INFORMATION}

Additional Supporting Information may be found online in the supporting information tab for this article. 